\title{
Identification of QTLs for relative root traits associated with phosphorus efficiency in two culture systems in Brassica napus
}

Article

Accepted Version

Wang, W., Zhang, Y., Ding, G., White, P. J., Broadley, M. R., Hammond, J. P., Jin, K., Cai, H., Xu, F. and Shi, L. (2019) Identification of QTLs for relative root traits associated with phosphorus efficiency in two culture systems in Brassica napus. Euphytica, 215 (11). 192. ISSN 0014-2336 doi: https://doi.org/10.1007/s10681-019-2512-4 Available at https://centaur.reading.ac.uk/87526/

It is advisable to refer to the publisher's version if you intend to cite from the work. See Guidance on citing.

To link to this article DOI: http://dx.doi.org/10.1007/s10681-019-2512-4

Publisher: Springer

All outputs in CentAUR are protected by Intellectual Property Rights law, including copyright law. Copyright and IPR is retained by the creators or other copyright holders. Terms and conditions for use of this material are defined in the End User Agreement. 


\section{CentAUR}

Central Archive at the University of Reading

Reading's research outputs online 
Identification of QTLs for relative root traits associated with phosphorus efficiency in two culture systems in Brassica napus

Wei Wanga,b, Ying Zhanga,b, Guangda Dinga,b, Philip J. White, Martin R. Broadleyd, John P. Hammonde,f, Kemo Jina,b, Hongmei Caib, Fangsen Xua,b, Lei Shia,b,*

aNational Key Laboratory of Crop Genetic Improvement, Huazhong Agricultural University, Wuhan 430070, China

bMicroelement Research Centre, Key Laboratory of Arable Land Conservation (Middle and Lower Reaches of Yangtze River), Ministry of Agriculture, Huazhong Agricultural University, Wuhan 430070, China

cThe James Hutton Institute, Invergowrie, Dundee DD2 5DA, UK

dPlant and Crop Sciences Division, School of Biosciences, University of Nottingham, Sutton Bonington Campus, Loughborough LE12 5RD, UK

eSchool of Agriculture, Policy and Development, University of Reading, Reading RG6 6AR, UK

fSouthern Cross Plant Science, Southern Cross University, Lismore NSW 2480, Australia

Wei Wang: weiwfftd2017@webmail.hzau.edu.cn

Ying Zhang: zhangying9629@126.com

Guangda Ding: dgd@mail.hzau.edu.cn

Philip J. White: philip.white@hutton.ac.uk

Martin R. Broadley: martin.broadley@nottingham.ac.uk

John P. Hammond: j.p.hammond@reading.ac.uk

Kemo Jin: kemo.jin@mail.hzau.edu.cn

Hongmei Cai: caihongmei@mail.hzau.edu.cn

Fangsen Xu: fangsenxu@mail.hzau.edu.cn

Lei Shi: leish@mail.hzau.edu.cn

*Correspondence: Lei Shi (leish@mail.hzau.edu.cn) 
Address: National Key Laboratory of Crop Genetic Improvement, Huazhong Agricultural University, Wuhan 430070, China

Tel: 0086-27-87286871

Fax: 0086-27-87280016

Abstract Modifications of root system morphology and architecture are considered important strategies of plant tolerance to phosphorus (P) deficiency. However, the effect of culture system on the responses of root traits to $\mathrm{P}$ deficiency is not well documented. In this study, the responses of root traits to $\mathrm{P}$ deficiency were recorded in a Brassica napus double haploid population consisting of 182 lines derived from a cross between cultivar 'Tapidor' and 'Ningyou 7' using an 'agar' system and a 'pouch and wick' system. Under P deficient conditions, more DH lines had greater total root length, primary root length, total lateral root length, mean lateral root length and less lateral root density in the 'pouch and wick' system than the 'agar' system. Ten and two quantitative trait loci (QTLs) were detected for the relative root traits in the 'agar' system and the 'pouch and wick' system, respectively. The QTL for the same trait in the 'agar' system did not overlap with that in the 'pouch and wick' system. Two QTL clusters identified in the 'agar' system were located on chromosome A09 (Cluster1 and Cluster2) and one on C04 (Cluster3), respectively. RLRN_A04b, RSDW_A09a and Cluster1 were found to affect the seed yield and/or yield-related traits in two field trials. Overall, this study demonstrated a significant impact of different culture systems on the responses of root traits to $\mathrm{P}$ deficiency and on the detection of QTLs for the relative root traits, and identified three major QTLs that could be employed for marker assisted selection of $\mathrm{P}$ efficient cultivars.

Keywords Root traits; Quantitative trait loci (QTLs); Phosphorus deficiency; 'agar' system; 'pouch and wick' system; Brassica napus

Abbreviations DH, double haploid; LP, a low phosphorus supply; LRD, lateral root density; LRL, total lateral root length; LRN, lateral root number; MLRL, mean lateral root length; OP, an optimal phosphorus supply; P, phosphorus; Pi, inorganic phosphate; PRL, primary root length; QTL, quantitative trait loci; RLRD, relative lateral root density; RLRL, relative total lateral root length; RLRN, relative lateral root number; RMLRL, relative mean lateral root length; RPRL, relative primary root length; RRFW, relative root fresh weight; RSDW, relative shoot dry weight; RTDW, relative total dry weight; RTRL, relative total root length; TRL, total root length 


\section{Introduction}

Phosphorus (P) is a component of cellular membranes as phospholipids, and is involved in multiple biological functions, such as energy transfer, photosynthesis, metabolic processes, intracellular signal transduction and gene replication and expression (Hawkesford et al. 2012). However, $50 \%$ of agricultural soils in the world are deficient in plant-available P, which leads to growth reduction, developmental delays, and severe crop failures (Lynch 2011; Elser 2012). In response to persistent $\mathrm{P}$ deficiency, plants have evolved a wide array of adaptive mechanisms to improve $\mathrm{P}$ acquisition efficiency and $\mathrm{P}$ utilization efficiency, including increased root/shoot ratio, modifications in root architecture to forage soil horizons for high phytoavailable $\mathrm{P}$, increased number and length of lateral roots and root hairs, the induction of high-affinity inorganic phosphate $(\mathrm{Pi})$ transporters, more exudation of acid phosphatases, organic acids or protons, symbiosis with arbuscular mycorrhizal (AM) fungi and change of metabolic processes (Hermans et al. 2006; Fita et al. 2011; Tian et al. 2012; Veneklaas et al. 2012; Haling et al. 2013; Lambers et al. 2013; White et al. 2013a, 2013b; Lapis-Gaza et al. 2014; López-Arredondo et al. 2014; Walder et al. 2015).

The alteration of root system architecture is a well-documented phenomenon in response to P starvation (Liao et al. 2004; Zhu et al. 2005a, 2005b; Wang et al. 2010; Bayuelo-Jiménez et al. 2011; Lambers et al. 2011, 2013; Lynch 2011). In the model plant Arabidopsis, root system architecture responses to $\mathrm{P}$ deficiency have been well characterized (White et al. 2005). Typically, a reduction of the primary root length (Williamson et al. 2001; Linkohr et al. 2002; López-Bucio et al. 2002; Svistoonoff et al. 2007) concomitantly associated with an increase in the number and length of lateral roots (Williamson et al. 2001; Linkohr et al. 2002; Al-Ghazi et al. 2003; López-Bucio et al. 2005; Nacry et al. 2005; Reymond et al. 2006) are observed in Pstarved Arabidopsis, but this root responses are largely genotype dependent. Compared with a P-rich medium, 37 of 73 Arabidopsis ecotypes showed both reduced primary root length and lateral root number at the P-poor medium, and $25 \%$ were affected in only one trait while the remaining accessions displayed no response to P availability (Chevalier et al. 2003), suggesting different physiological strategies are exploited to adapt to P deficiency within a species. 
Additionally, the growth medium has strong effect on the root responses to P deficiency. For example, when grown in P-deficient nutrient solution, most of the tested rice genotypes formed longer root hairs, but many of these rice varieties tended to produce shorter root hairs in an upland field with a low P supply (Nestler and Wissuwa 2016).

Two genes have been cloned for the modifications of the root traits response to P starvation by forward genetics (Svistoonoff et al. 2007; Gamuyao et al. 2012). In Arabidopsis, Low Phosphate Root1 (LPR1) encoding a multicopper oxidase (MCO) functionally plays an important role in primary root development in response to $P$ deficiency (Svistoonoff et al. 2007). In rice, phosphorus-starvation tolerance 1 (PSTOL1) was identified to regulate the early crown root development and root proliferation at a low P supply (Gamuyao et al. 2012).

Traits related to P efficiency in plants are generally divided into single traits and relative traits (Wang et al. 2018). Relative traits are calculated as the quotient of the value of a trait observed when plants are grown at a reduced P supply divided by the value of the trait when plants are grown with optimal $\mathrm{P}$ nutrition. These include the $\mathrm{P}$ efficiency coefficient (i.e. the ratio of biomass at the seedling stage or grain yield at maturity in plants grown with a low versus an optimal P supply) which has been used to evaluate tolerance to P deficiency in oilseed rape (Duan et al. 2009) and rice (Ni et al. 1998; Ming et al. 2000). Although single traits are more commonly used in quantitative trait loci (QTLs) mapping studies for P efficiency traits and in breeding programs, relative traits indicate the tolerance of a genotype to reduced P availability. Thus the co-located QTLs both for a single trait and for a relative trait should be more useful than the QTLs only for a single trait in the breeding of P efficient cultivars (Wang et al. 2018).

Oilseed rape (Brassica napus L.) is commonly used to produce cooking oil for human consumption, fodder for animal feeds and renewable feedstock for biodiesel production (Liu et al. 2015). Despite the many QTLs for P-efficiency related traits that have been identified at the seedling stage (Yang et al. 2010, 2011; Shi et al. 2013a; Zhang et al. 2016; Wang et al. 2017) and mature stage (Ding et al. 2012; Shi et al. 2013b), no QTL for P efficiency has been cloned and functionally characterized in Brassica napus (B. napus) so far. In this study, the genetic variations of the root morphological traits in a double haploid (DH) population of B. napus (BnaTNDH population) derived from a cross between Tapidor and Ningyou 7 were investigated 
at a low and an optimal P supply with an 'agar' system and a 'pouch and wick' system. Ningyou 7 was found to have a higher seed yield than that of Tapidor at a low P supply in both pot culture and field trials (Shi et al. 2010, 2013b). The relative root traits were employed to identify QTLs for the plasticity of root traits in response to P deficiency. These will contribute to the understanding of the effect of growth environments on the seedling root traits responding to $\mathrm{P}$ deficiency and their QTLs.

\section{Materials and methods}

\section{Plant materials}

The BnaTNDH mapping population consisted of 182 lines, which was generated through anther culture of the $\mathrm{F}_{1}$ generation of a cross between B. napus cultivar Tapidor and Ningyou 7 (Qiu et al. 2006).

\section{High throughput phenotyping and data analysis}

In the 'agar' system, the root traits and biomass traits of the BnaTNDH population and the parents had been screened previously at a Pi concentration of $0 \mathrm{mM}$ (a low phosphorus supply, LP) and $0.625 \mathrm{mM}$ (an optimal phosphorus supply, OP), respectively (Shi et al. 2013a). Briefly, surface sterilized seeds were sown into vented polystyrene trays (QTray; $240 \times 240 \times 20 \mathrm{~mm}$; Molecular Devices, Hampshire, UK) containing $300 \mathrm{~mL}$ 0.8\% (w/v) agar and a modified basal salt mix (Murashige and Skoog 1962) with either OP added as $\mathrm{KH}_{2} \mathrm{PO}_{4}$ or LP, with $0.625 \mathrm{mM}$ $\mathrm{KCl}$ added to provide $\mathrm{K}$. Seeds were sown $3 \mathrm{~cm}$ from the top edge of the tray, with four seeds per line and two lines per tray. Trays were sealed with Nescofilm and placed $10^{\circ}$ from vertical in a growth room under a 16 -h photoperiod at a constant temperature of $24{ }^{\circ} \mathrm{C}$. Illumination was provided by a bank of 84 100-W cool fluorescent tubes (Philips, Eindhoven, Netherlands), giving a photon flux density between 400 and $700 \mathrm{~nm}$ of $80-100 \mu$ mol photons $\mathrm{m}-2 \mathrm{~s}-1$ at plant height. For each line, 16 seeds were sown across four independent replicates, at both LP and 
OP. Trays were placed randomly within the growth room. Images of the root systems were captured using a flatbed scanner (Scanjet 3670; Hewlett-Packard, Palo Alto, CA, USA) 12 d after sowing. At harvest, shoot and root fresh weight were determined, respectively. Tissue samples were dried at $80^{\circ} \mathrm{C}$ and dry weights (shoot dry weight; root dry weight) determined. Images were loaded into ImageJ (Abràmoff et al. 2004). Primary root length (PRL) and total lateral root length (LRL) were measured. Lateral root numbers (LRN) were counted and used to calculate lateral root density (LRD, LRN/PRL) and mean lateral root length (MLRL, LRL/LRN). Total root length (TRL) was calculated as the sum of PRL and LRL. Raw data were entered into GenStat (15th Edition, VSN International Ltd, Hemel Hempstead, UK). To acquire adjusted line means, the REML (residual maximum likelihood) procedure was performed using the $\left([\mathrm{P}]_{\mathrm{ext}}+\right.$ Line $+[\mathrm{P}]_{\mathrm{ext}} \times$ Line) term as a fixed factor and (Replicate + Replicate/Run + Replicate/Run/Plate + Replicate/Run/Plate/Position) as a random factor.

In the 'pouch and wick' system, the root traits of the BnaTNDH population and the parents had also been investigated previously at a Pi concentration of $0 \mathrm{mM}(\mathrm{LP})$ and $0.25 \mathrm{mM}(\mathrm{OP})$, respectively (Zhang et al. 2016). Briefly, this system comprised growth pouches assembled from blue germination paper (SD7640; Anchor Paper Company, St Paul, MN, USA), re-cut to $24 \times 30 \mathrm{~cm}$ and overlain with black polythene (Cransford Polythene Ltd, Woodbridge, UK). Along their shorter edges, the paper and polythene were clipped together using 'bulldog'-type fold-back clips to each side of an acrylic bar (Acrylic Online, Hull, UK) giving 2 germination papers per pouch. The growth pouches were suspended above plastic drip trays containing a $1 / 4$ strength Hoagland's solution (No. 2 Basal Salt Mixture, Sigma Aldrich, Dorset, UK) with either OP added as $\mathrm{KH}_{2} \mathrm{PO}_{4}$ or LP, with $0.125 \mathrm{mM} \mathrm{K}_{2} \mathrm{SO}_{4}$ added for balanced $\mathrm{K}$, supported within lightweight aluminium/polycarbonate frames. A single seed was sown in the middle of the upper edge of each germination paper by pressing the seed into the paper. Each genotype was grown in one experimental run under a $12 \mathrm{~h}$ photoperiod with $18 / 15^{\circ} \mathrm{C}$ day/night temperatures and relative humidity of $60-80 \%$, and pouches were randomly allocated to a position within each column of each tank, giving $\sim 24$ replicates per run. Photosynthetically Active Radiation (PAR; measured at plant height with a 190 SB quantum sensor; LI-COR Inc., Lincoln, NE, USA) was $207 \mu \mathrm{mol} \mathrm{m-2} \mathrm{s-1,} \mathrm{generated} \mathrm{by} 400 \mathrm{~W}$ white fluorescent lamps (HIT 400w/u/Euro/4K, 
Venture Lighting, Rickmansworth, UK). Drip trays were replenished with $500 \mathrm{~mL}$ of deionized water every $3 \mathrm{~d}$. Fourteen days after sowing, the polythene sheets were removed from all pouches and images were taken using a Digital Single Lens Reflex (DSLR) camera (Canon EOS 1100D, Canon Inc., Tokyo, Japan) with a focal length of $35 \mathrm{~mm}$ at a fixed height of 75 $\mathrm{cm}$. The root images were cropped by reducing extraneous pixels on bulked images, using XnConvert (Version 1.66, www.xnconvert.com). Cropped images were analysed using RootReader2D (RR2D). PRL, LRL and LRN were automatically calculated by RR2D. LRD was calculated as the ratio of LRN to PRL, and MLRL was calculated as the ratio of LRL to LRN. TRL was calculated as the sum of PRL and LRL. A random term $[($ Run/Frame/Column/Position/Paper side $)+([\mathrm{P}]$ ext $\times$ Line $)]$ except for a fixed factor (Line) was used to estimate line means with the REML procedure.

The relative root traits and relative biomass traits of each line were estimated as quotient of the mean value of a trait at LP divided by the mean value of the trait at OP. The plasticity of root traits in response to $\mathrm{P}$ deficiency were calculated using the following formula: plasticity $=$ (mean value of a trait at LP - mean value of a trait at OP) / mean value of a trait at OP. The correlation coefficients among these traits were computed using the Pearson's correlation method of SPSS/WIN 18.0 program.

\section{QTL mapping}

A SNP-based high-density BnaTNDH genetic map comprising 2041 markers (Zhang et al. 2016) was used for the QTL mapping. The composite interval mapping (CIM) program of WinQTLCart v2.5 (Wang et al. 2011) was used to detect significant QTLs for relative traits of the BnaTNDH population. The number of control markers, window size and walking speed were set to 5, $10 \mathrm{cM}$ and $1 \mathrm{cM}$, respectively. The backward regression algorithm was used to obtain cofactors. Empirical threshold for each trait was computed using the permutation test (1,000 permutations, overall error level 5\%) for CIM (Churchill and Doerge 1994). The estimated additive effect and the percentage of phenotypic variation explained by each putative QTL were obtained using the CIM model. The confidence intervals were set as the map interval 
that corresponded to a 2-LOD decline on either side of the LOD peak.

The epistatic QTLs were identified for the relative root traits by the QTL IciMapping v4.1 (Meng et al. 2015), which is public and freely available (http://www.isbreeding.net/software/). The epistatic QTLs were detected by the ICIM-EPI method using single environment phenotypic values. The $P$ values for entering variables (PIN) and removing variables (POUT) were set at 0.0001 and 0.0002 , respectively, and the scanning step was $5 \mathrm{cM}$. The LOD threshold for the epistatic QTL was set as the default manual input value of the software. The proportion of observed phenotypic variance explained by each epistatic QTL and the corresponding additive effects were also estimated.

\section{Identification and integration of the QTL clusters}

A QTL cluster was defined as two or more significant QTLs with overlapping confidence interval. Individual QTLs for relative root traits and relative biomass traits in a QTL cluster were integrated in a meta-analysis using BioMercator v4.2 (Arcade et al. 2004). Meta-analysis computing is based on the position of each input QTL, and on the variance of this position, assessed through confidence interval values. The algorithm developed by Goffinet and Gerber (2000) was employed to conduct the QTL meta-analysis, and the model with lowest Akaike value was selected for QTL integration. The principle of integration is that the confidence interval of integrated QTL should contain the peak position of component QTLs. The integrated QTL for each QTL cluster was mapped to the reference genome (Darmor- $b z h$ ) according to the physical position of the two flanking markers. The available reference genome of B. napus (Chalhoub et al. 2014) and the functional annotation of the Arabidopsis genome (https://www.arabidopsis.org/) were employed for the prediction of putative candidate genes. 


\section{Results}

Difference in root traits of BnaTNDH population in respond to Pi starvation between 'agar' system and 'pouch and wick' system

When compared with OP, more DH lines had greater TRL, PRL, LRL and MLRL in the 'pouch and wick' system than the 'agar' system at LP (Fig. 1a-d). Accordingly, the mean plasticity of TRL, PRL, LRL, and MLRL of the BnaTNDH population in the 'pouch and wick' system was larger than that in the 'agar' system (Supplementary Fig. 1a-d). Nearly $65.0 \%$ of the DH lines had an increase in LRN at LP compared with OP in both the 'agar' and 'pouch and wick' systems, while the mean plasticity of LRN of the BnaTNDH population in the 'pouch and wick' system was $12.6 \%$ greater than that in the 'agar' system (Fig. 1e; Supplementary Fig. 1e). Nearly $60 \%$ of lines that showed increased LRN at LP were the same in both culture systems. In 'agar' system, the LRD of $90.0 \%$ of the DH lines was increased at LP as compared with OP and the mean plasticity of LRD of the BnaTNDH population was $59 \%$. While in the "pouch and wick' system, the LRD of $75.4 \%$ of the DH lines was increased at LP and the mean plasticity of LRD of the BnaTNDH population was only 14.1\% (Fig. 1f; Supplementary Fig. 1f). Similarly, when compared with OP, the TRL, PRL, LRL and MLRL of cultivars Tapidor and Ningyou 7 was increased at LP in the 'pouch and wick' system but decreased in the 'agar' system, while the LRN and LRD of cultivars Tapidor and Ningyou 7 was increased at LP in both the 'agar' system and the 'pouch and wick' system (Fig. 1).

Phenotypic variation and correlation among relative root traits in the 'agar' and 'pouch and wick' systems

A wide range of variation was observed in all the relative traits among the BnaTNDH lines in both culture systems (Table 1; Fig. 2). Values of the six relative root traits of Tapidor were all higher than that of Ningyou 7 in both culture systems, except relative primary root length (RPRL) in both culture systems and relative mean lateral root length (RMLRL) in the 'agar' 
system (Table 1; Fig. 2). The mean of all the relative traits, except for the relative lateral root density (RLRD), of BnaTNDH population and both parental lines was higher in the 'pouch and wick' system than that in the 'agar' system (Table 1). Moreover, larger coefficients of variation (CVs) of these traits, except for RLRD, of BnaTNDH population were observed in the "pouch and wick' system compared with that in the 'agar' system (Table 1). In both culture systems, the frequency distribution of all the traits showed continuous phenotypic variation, and significant transgressive segregations were observed in the population (Table 1; Fig. 2).

Pearson's correlation coefficients between relative root traits were calculated (Supplementary Table 1). Significant positive correlations between relative total root length (RTRL) and the other five relative root traits of BnaTNDH population were observed in both culture systems. Of these, the correlation of RTRL and relative total lateral root length (RLRL) in the 'pouch and wick' system $(r=0.93 ; P<0.001)$ was much larger than that in the 'agar' system $(r=0.53 ; P<0.001)$. RPRL and RLRL were significantly correlated in both the 'agar' $(r$ $=0.27 ; P<0.001)$ and 'pouch and wick' systems $(r=0.49 ; P<0.001)$. There was a significant positive correlation between RPRL and relative lateral root number (RLRN) in the 'pouch and wick' system $(r=0.80 ; P<0.001)$, while there was no correlation between them in the 'agar' system $(r=0.06)$. In the 'agar' system, a significant negative correlation was observed between the 'agar' and 'pouch and wick' systems. There was a significant positive correlation between RLRL and RLRD in the 'pouch and wick' system $(r=0.35 ; P<0.001)$, while the correlation RPRL and RLRD $(r=-0.39 ; P<0.001)$, but no correlation was observed in the 'pouch and wick' system $(r=0.06 ; \mathrm{P}=\$ \$ \$$ ). RLRL was significantly correlated with RMLRL and RLRN in both was not significant in the 'agar' system $(r=0.05)$. There was no correlation between RMLRL and RLRN in the 'pouch and wick' system $(r=0.01)$, but a weak negative correlation was observed in the 'agar' system $(r=-0.17 ; P<0.05)$. A strong positive correlation and a moderate positive correlation were observed between RLRN and RLRD in the 'agar' system $(r=0.83$; $P<0.001)$ and the 'pouch and wick' system $(r=0.47 ; P<0.001)$, respectively. Moreover, in the 'agar' system, the relative biomass traits were significantly correlated with relative root traits (Supplementary Table 2), such as between relative total dry weight (RTDW) and RTRL ( $r=$ $0.65 ; P<0.001)$, and between relative shoot dry weight (RSDW) and RPRL $(r=0.52 ; P<0.001)$. 
However, no correlation was observed for the same trait between the two culture systems (Supplementary Table 1).

QTLs for relative root and biomass traits of BnaTNDH mapping population in the 'agar' and 'pouch and wick' systems

A QTL analysis was performed to identify the genetic factors responsible for the relative root traits in both the 'agar' and the 'pouch and wick' systems. In the 'agar' system, a total of 10 significant QTLs were identified for six relative root traits across six of the 19 chromosomes (Supplementary Table 3). Among them, one QTL for RTRL, one for RPRL, one for RLRL, one for RMLRL and one for RLRD were mapped on chromosomes A09, A08, A09, A07 and C04, respectively, accounting for $7.4 \%-12.8 \%$ of the phenotypic variation. Five QTLs for RLRN were mapped on A04, C04 and C08, which jointly explained $46.7 \%$ of the phenotypic variation. With the exception of one QTL on A07 for RMLRL (RMLRL_A07), all the QTLs for relative root traits had a negative additive effect (Supplementary Table 3). The alleles from Tapidor increased the values of all the relative root traits except for RMLRL. Moreover, two QTLs for RTDW, two for RSDW, one for relative shoot fresh weight on chromosome A09, and two for relative root fresh weight (RRFW) on both chromosome A09 and C09 were detected, respectively, which explained $7.9 \%-15.3 \%$ of the phenotypic variation (Supplementary Table 3). Among these nine QTLs, the alleles of seven QTLs from Tapidor contributed to the increase of relative traits except for two QTLs on chromosome C09 for RRFW (RRFW_C09a, RRFW_C09b) (Supplementary Table 3). In the 'pouch and wick' system, one QTL for RPRL and one for RLRL were detected on chromosomes A03 and C04, respectively, and no QTLs were identified for RTRL, RMLRL, RLRN and RLRD with the BnaTNDH mapping population (Supplementary Table 3). The QTL for the same trait in the 'agar' system did not overlap with that in the 'pouch and wick' system, which was consistent with the poor correlation of these traits between the two culture systems among genotypes.

Epistatic interaction analysis was conducted with the ICIM approach using phenotypic values from the 'agar' and 'pouch and wick' systems independently (Supplementary Table 4). 
In the 'agar' system, one epistatic QTL was identified for RTRL, accounting for 3.6\% of the phenotypic variation. In the 'pouch and wick' system, there was one epistatic QTL controlling RPRL and another one controlling RLRD, which explained $4.9 \%$ and $13.2 \%$ of the phenotypic variation, respectively. These three epistatic QTLs had a negative effect of additive by additive interaction, indicating that two loci from different parental lines take the positive effects (Supplementary Table 4).

\section{QTL clusters identified in the 'agar' system}

Two QTL clusters identified in the 'agar' system were located on chromosome A09 (Cluster1 and Cluster2) and one was located on C04 (Cluster3) (Fig. 3; Supplementary Table 5). Cluster1 contained one QTL for RTRL, one for RTDW, and one for RRFW. Four QTLs controlling RTDW, RSDW, relative shoot fresh weight and RRFW were co-located in Cluster2. In Cluster3, a QTL associated with RLRN was co-located with a QTL for RLRD (Fig. 3; Supplementary Table 5). The average LOD score of the component QTLs in these three QTL clusters ranged from 5.03-5.35, and each cluster accounted for $11.2 \%-12.2 \%$ of the average phenotypic variation (Supplementary Table 5). The confidence intervals of Cluster1, Cluster2 and Cluster3 were defined as 129.5-131.3, 135.9-138.1 and 30.9-32.9 cM, respectively, using BioMercator v4.2 by QTL meta-analysis (Supplementary Table 5).

Co-located QTLs for the relative root traits and for the single root traits or the seed yieldrelated traits

We have mapped the significant QTLs for the single root traits of the BnaTNDH population at LP and OP in the 'agar' system (Shi et al. 2013a), in the 'pouch and wick' system (Zhang et al. 2016), and the seed yield and yield-related traits in the field trials (Shi et al. 2013b). These QTLs had been summarized in Supplementary Table 6 and Supplementary Table 7. In the 'agar' system, the average number of QTLs detected for each single root trait was 2.7 at LP and 2.3 at OP, while the average number of QTLs detected for each relative root trait was 1.7. In the 
'pouch and wick' system, the average number of QTLs detected for each single root trait was 3.7 at LP and 1.7 at OP, while the average number of QTLs detected for each relative root trait was only 0.3 . Therefore, the QTLs for the relative root traits were less than that for the single root traits. In the 'agar' system, two of the ten QTLs for the relative root traits co-located with the QTL for respective single root trait at OP, including RMLRL_A07 and RLRN_C08 (Supplementary Fig. 2). In the 'pouch and wick' system, RPRL_A03 (one of the two QTLs for relative root traits) was found to overlap with the QTL for its single root trait at OP (Supplementary Fig. 2).

Seven QTLs associated with the relative root trait and/or relative biomass trait were colocated with the QTLs for seed yield and yield-related traits (Table 2). Among these QTLs, RLRN_A04b, RSDW_A09a and Cluster1 were found to affect the seed yield and yield-related traits in two of three field trials (Table 2). RLRN_A04b was co-located with the QTL for seed weight of 1,000 seeds at OP $\left(\mathrm{P}_{2} \mathrm{O}_{5}, 90 \mathrm{Kg}\right.$ ha-1) in Tri.1 (field trial conducted from Sept 2008 to May 2009), and at $\mathrm{LP}\left(\mathrm{P}_{2} \mathrm{O}_{5}, 9 \mathrm{Kg}\right.$ ha-1) and $\mathrm{OP}\left(\mathrm{P}_{2} \mathrm{O} 5,90 \mathrm{Kg}\right.$ ha-1) in Tri.2 (field trial conducted from Sept 2009 to May 2010). RSDW_A09a was co-located with the QTL for seed yield per hectare at $\mathrm{LP}\left(\mathrm{P}_{2} \mathrm{O}_{5}, 9 \mathrm{Kg}\right.$ ha-1) and plant height at $\mathrm{OP}$ in Tri.1, and for height to the first primary branch at OP in Tri.2. Cluster1 was co-located with the QTL for seed yield per hectare at LP in Tri.1, and for height to the first primary branch, plant height, seed yield per hectare at OP in Tri.2. Additionally, RSDW_A09a, and Cluster1 and Cluster2 were co-located with QTLs SY_LP_A09a $\left(\mathrm{R}_{2}=4.5 \%\right)$ and SY_LP_A09b $\left(\mathrm{R}_{2}=5.8 \%\right)$ associated with seed yield based on best linear unbiased estimation (BLUE) across three field trials at LP, respectively (Supplementary Table 8). In the genomic regions of RLRN_A04b (17364075-17578367 bp), RSDW_A09a (32624626-32900779 bp) and Cluster1 (32900851-33338388 bp), there were 53, 60 and 97 annotated genes, respectively (Supplementary Table 9). In RLRN_A04b and RSDW_A09a, there seemed to be no annotated genes known to be involved in tolerance to P deficiency. In Cluster1, BnaA09g50010D is orthologous to AT1G06160 (ERF59) in Arabidopsis, which encodes a member of the ERF (ethylene response factor) subfamily B-3 of ERF/AP2 transcription factor family. 
Difference in the root plasticity of $B$. napus in response to $P$ deficiency between 'agar' system and 'pouch and wick' system

Phosphorus plays a critical role in all major developmental processes and reproduction in plants, including seed germination, seedling growth, flower initiation and seed formation (Hawkesford et al. 2012). P deficiency in soil is one of the major limiting factors for crop production throughout the world (Lynch 2007; Veneklaas et al. 2012). Root system architecture traits are vital for soil exploration and nutrient acquisition (Lynch 2007). The remodeling of root morphology and architecture is the most evident change in response to P deficiency, which provides a shallower growth angle of axial roots for obtaining P in the top part of soils (Lynch 2007; Liang et al. 2014), the proteoid roots (cluster roots: dense clusters of short side roots) releasing carboxylates for mobilising $\mathrm{P}$ to improve soil $\mathrm{P}$ availability (Shane and Lambers 2005; Lambers et al. 2011, 2013), and/or an increase in number and length of lateral roots and root hairs for enlarging the root surface area scavenging for P in soils (Jain et al. 2007; Lynch 2011; Haling et al. 2013; Niu et al. 2013). In this study, there were significant differences in the response of root traits of B. napus to P starvation between an 'agar' system and a 'pouch and wick' system (Fig. 1; Supplementary Fig. 1). At LP compared with OP, there was a decrease in TRL of most DH lines and both parental lines in the 'agar' system, which was caused by a reduction in both PRL and LRL, while an increased TRL was observed in the 'pouch and wick' system. A reduced PRL is a widely reported physiological response in P-deficient Arabidopsis grown on vertical agar plates (Williamson et al. 2001; Linkohr et al. 2002; Al-Ghazi et al. 2003; López-Bucio et al. 2005; Nacry et al. 2005; Reymond et al. 2006; Ward et al. 2008; Müller et al. 2015; Mora-Macías et al. 2017) and is possibly the result of iron (Fe) toxicity (Ward et al. 2008). The reduced PRL of B. napus at LP compared to OP in the 'agar' system is consistent with these studies on Arabidopsis. However, the reduction in LRL of B. napus at LP compared to OP in the 'agar' system contrasts with an increase in LRL observed in Arabidopsis grown on agar plates at LP compared to OP (Williamson et al. 2001; Linkohr et al. 2002; Al-Ghazi et al. 
2003; López-Bucio et al. 2005; Nacry et al. 2005; Reymond et al. 2006). The P-deficient $B$. napus plants grown in the 'pouch and wick' system seemed to have greater PRL, which is consistent with studies of plants grown in nutrient solutions (Zhang 2009; Wang et al. 2017). The LRL of B. napus grown in the 'pouch and wick' system was greater at LP than at OP, which is consistent with studies of Brassica oleracea grown on vertical glass plates supported on blue blotter paper (Hammond et al. 2009).

Under P-limited conditions, an increased LRN was observed in both culture systems (Fig. 1e; Supplementary Fig. 1e). Enhanced lateral root formation at LP has been reported for Arabidopsis grown on vertically oriented agar plates (López-Bucio et al. 2002; SánchezCalderón et al. 2006; Péreztorres et al. 2008), Brassica oleracea grown on vertical glass plates supported on blue blotter paper (Hammond et al. 2009) and oilseed rape grown hydroponically (Zhang et al. 2011). The reduction in MLRL at LP compared to OP in the 'agar' system contrasts with an increase in MLRL at LP compared to OP in the 'pouch and wick' system (Fig. 1d; Supplementary Fig. 1d). A significant increase in LRD at LP was found in both culture systems (Fig. 1f; Supplementary Fig. 1f). However, the LRD in the 'agar' system had a significantly greater plasticity than that in the 'pouch and wick' system, mainly because of a large reduction of PRL in the 'agar' system (Fig. 1b; Supplementary Fig. 1b).

The response of roots to unilateral light is a species-dependent and can include positive phototropism, negative phototropism and no phototropism (Hubert and Funke 1937; Kutschera and Briggs 2012). The roots of Arabidopsis display a negative phototropic response (Boccalandro et al. 2008; Kutschera and Briggs 2012). Negative root phototropism prevents light stress in the upper layers of the soil where light penetration is greatest, reduced desiccation phenomena, and enhanced seedling survival under dry and windy conditions by mediating plastic increases in the efficiency of root growth near the soil surface (Galen et al. 2004, 2006; Kutschera and Briggs 2012). In this study, the root system was exposed to light in the 'agar' system (Shi et al. 2013a), while polythene sheets were employed to cover the root in the "pouch and wick' system (Zhang et al. 2016). Increased PRL and LRL were generally observed in Pdeficient B. napus plants in the 'pouch and wick' system, while the situation in the 'agar' system was opposite. Moreover, when oilseed rape seedlings were grown in P-deficient nutrient 
solution with roots in dark, the TRL and PRL were both enhanced compared with plants in Preplete nutrient solution (Zhang 2009; Zhang et al. 2011; Wang et al. 2017). These observations suggest that shielding roots from light reduced the sensitivity of root system elongation to $\mathrm{P}$ deficiency in B. napus. In Arabidopsis, plants with roots in darkness had longer PRL and more LRN than plants with roots exposed to light conditions under P sufficient conditions (SilvaNavas et al. 2016). P deficiency significantly inhibited the elongation of the primary root of Arabidopsis and B. napus cultivars when roots were exposed to light, but had no effect when roots grew in darkness (Supplementary Figs. 3-5). Similarly, in Arabidopsis, roots grown in darkness showed less sensitivity to nitrogen deficiency and salt stress compared with those exposed to light (Silva-Navas et al. 2015). The increased number of lateral roots under $\mathrm{P}$ deficient conditions happened in both the 'agar' and 'pouch and wick' systems, indicating that the increase in LRN is not light-sensitive.

Plants grown in 'agar' and 'pouch and wick' systems can be used to remove the influence of complex soil environment on root growth. However, in agricultural system, natural soils exhibit considerable spatial and temporal variability in structure and resource availability (Jin et al. 2017). Since significant $G \times E$ interaction occur for root system architecture in the field (White et al. 2013b), only a few QTLs for the seed yield and yield-related traits of the BnaTNDH population investigated in the field (Shi et al. 2013b) co-located with the QTLs for the root or relative root traits investigated by 'agar' and 'pouch and wick' systems. Some promising technologies, such as X-ray computed tomography or magnetic resonance imaging have been developed for visualizing plant root systems within their natural soil environment noninvasively (Metzner et al. 2015) and may prove useful in the future for identifying QTL associated with root traits in response to abiotic factors.

\section{QTLs for relative root traits of B. napus}

Relative root traits were used to evaluate the root plasticity of B. napus in response to $\mathrm{P}$ deficiency in this study. Considerable transgression of six relative root traits of B. napus were observed in both the 'agar' and 'pouch and wick' culture systems (Table 1; Fig. 2), indicating 
that both parental lines carry genes with alleles contributing to an increase or a decrease of the relative root traits. The culture system had a significant influence on the relative root traits of two parental lines, and greater differences in all relative root traits except RLRN were observed between the two parental lines in the 'pouch and wick' system than in the 'agar' system (Table 1). Pairs of relative root traits, such as RTRL and RPRL, RTRL and RLRL, RLRL and RMLRL, were significantly correlated across the two culture systems (Supplementary Table 1), which is consistent with the correlation between TRL and PRL, TRL and LRL, LRL and MLRL (Zhang et al. 2016). However, correlations between some pairs of relative traits, e.g. RPRL and RLRN, RPRL and RLRD were not stable across the two culture systems (Supplementary Table 1), suggesting that there are different $\mathrm{P}$ deficiency-induced modulations of root system architecture in the two culture systems.

There was a difference in the genetic control of the relative root traits between plants grown in the 'agar' and 'pouch and wick' systems. One QTL for RTRL, one for RPRL, one for RLRL, one for RMLRL, one for RLRD, and five QTLs for RLRN were identified in the 'agar' system, while only one QTL for RPRL and one for RLRL were detected in the 'pouch and wick' system (Fig. 3; Supplementary Table 3). The QTLs identified for the same trait in the two culture systems were not co-located, which could account for the poor correlations among genotypes for the same traits studied in the two culture systems (Supplementary Table 1). The different genetic control of the relative root traits between plants grown in the 'agar' and 'pouch and wick' systems indicates that the plasticity of root system architecture responding to P deficiency is largely influenced by environmental factors like the light in this study. Larger coefficients of variation (CVs) of RTRL, RMLRL and RLRN were observed in the 'pouch and wick' system compared to the 'agar' system (Table 1), but no QTL was discovered for these traits in the 'pouch and wick' system. Thus, the trait variation may be not a good indicator for the number of QTLs that could be identified (Ghandilyan et al. 2009).

The QTLs for the relative root traits, RPRL_A03, RMLRL_A07 and RLRN_C08, co-located with the QTLs for the respective single root traits at OP (Supplementary Fig. 2), implying that these three QTLs only affected their respective single root trait at OP. The co-located QTLs both for a relative root trait and for a single root trait at LP should be more useful than the QTLs 
only for a relative root trait in the breeding of P efficient cultivars. In this study, RLRL_C04 was discovered to overlap with a significant SNP (Bn-scaff_15712_8-p121295) for LRL at LP identified by genome-wide association studies (GWAS) in the 'pouch and wick' system (Wang et al. 2017), which may play an important role in lateral root growth and development in response to $\mathrm{P}$ deficiency at seedling stage.

Three QTLs for the relative root traits were identified to affect the seed yield and yieldrelated traits in two of three field trials (Table 2). In the intervals of QTLs RLRN_A04b and RSDW_A09a, no annotated genes were involved in the response of plant to P deficiency, which indicated that there were novel genes in these QTL regions or several annotated genes had novel function associated with P deficiency response. In Cluster1, BnaA09g50010D (homologous to $A T 1 G 06160$ ) was predicted as a promising candidate gene. AT1G06160 (ERF59) is a member of the ERF (ethylene response factor) subfamily B-3 of ERF/AP2 transcription factor family (Nakano et al. 2006). ERF1 and ERF2 have been demonstrated to regulate the root growth of Arabidopsis and rice, respectively (Mao et al. 2016; Xiao et al. 2016). The candidate gene underlying the three target QTLs can be identified by investigating the phenotype of the Arabidopsis mutant at LP and OP, or by developing near-isogenic lines to allow further fine mapping of these QTLs and the cloning of potential candidate genes.

\section{Acknowledgements}

This research was supported by the National Key R\&D Program of China (Grant No. 2017YFD0200200), the Applied Basic Research Fronts Program of Wuhan city (2018020401011302), National Nature Science Foundation of China (31972498), Fundamental Research Funds for the Central Universities of China (Grant No. 2662019PY013) and the Rural and Environment Science and Analytical Services Division (RESAS) of the Scottish Government.

\section{Compliance with ethical standards}

\section{Conflict of interest}

The authors declare that they have no competing interests. 


\section{References}

529

530

531

532

533

534

535

536

537

538

539

540

541

542

543

544

545

546

547

548

549

550

551

552

553

554

555

556

557

558

559

560

561

562

Abràmoff MD, Magalhães PJ, Ram SJ (2004) Image processing with ImageJ. Biophoton Int $11: 36-42$

Al-Ghazi Y, Muller B, Pinloche S, Tranbarger TJ, Nacry P, Rossignol M, Tardieu F, Doumas P (2003) Temporal responses of Arabidopsis root architecture to phosphate starvation: evidence for the involvement of auxin signalling. Plant Cell Environ 26:1053-1066

Arcade A, Labourdette A, Falque M, Mangin B, Chardon F, Charcosset A, Joets J (2004) BioMercator: integrating genetic maps and QTL towards discovery of candidate genes. Bioinformatics 20:2324-2326

Bayuelo-Jiménez JS, Gallardo-Valdéz M, Pérez-Decelis VA, Magdaleno-Armas L, Ochoa I, Lynch JP (2011) Genotypic variation for root traits of maize (Zea mays L.) from the Purhepecha Plateau under contrasting phosphorus availability. Field Crops Res 121:350362

Boccalandro HE, De Simone SN, Bergmann-Honsberger A, Schepens I, Fankhauser C, Casal JJ (2008) PHYTOCHROME KINASE SUBSTRATE1 regulates root phototropism and gravitropism. Plant Physiol 146:108-115

Chalhoub B, Denoeud F, Liu S, Parkin IA, Tang H, Wang X, Chiquet J, Belcram H, Tong C, Samans B, Corréa M, Da Silva C, Just J, Falentin C, Koh CS, Le Clainche I, Bernard M, Bento P, Noel B, Labadie K, Alberti A, Charles M, Arnaud D, Guo H, Daviaud C, Alamery S, Jabbari K, Zhao M, Edger PP, Chelaifa H, Tack D, Lassalle G, Mestiri I, Schnel N, Le Paslier MC, Fan G, Renault V, Bayer PE, Golicz AA, Manoli S, Lee TH, Thi VH, Chalabi S, Hu Q, Fan C, Tollenaere R, Lu Y, Battail C, Shen J, Sidebottom CH, Wang X, Canaguier A, Chauveau A, Bérard A, Deniot G, Guan M, Liu Z, Sun F, Lim YP, Lyons E, Town CD, Bancroft I, Wang X, Meng J, Ma J, Pires JC, King GJ, Brunel D, Delourme R, Renard M, Aury JM, Adams KL, Batley J, Snowdon RJ, Tost J, Edwards D, Zhou Y, Hua W, Sharpe AG, Paterson AH, Guan C, Wincker P (2014) Early allopolyploid evolution in the post-Neolithic Brassica napus oilseed genome. Science 345:950-953

Chevalier F, Pata M, Nacry P, Doumas P, Rossignol M (2003) Effects of phosphate availability on the root system architecture: large-scale analysis of the natural variation between Arabidopsis accessions. Plant Cell Environ 26:1839-1850

Churchill GA, Doerge RW (1994) Empirical threshold values for quantitative trait mapping. Genetics 138:963-971

Ding G, Zhao Z, Liao Y, Hu Y, Shi L, Long Y, Xu F (2012) Quantitative trait loci for seed 
yield and yield-related traits, and their responses to reduced phosphorus supply in Brassica napus. Ann Bot 109:747-759

Duan HY, Shi L, Ye XS, Wang YH, Xu FS (2009) Identification of phosphorous efficient germplasm in oilseed rape. J Plant Nutr 32:1148-1163

Elser JJ (2012) Phosphorus: a limiting nutrient for humanity? Curr Opin Biotechnol 23:833838

Fita A, Nuez F, Picó B (2011) Diversity in root architecture and response to P deficiency in seedlings of Cucumis melo L. Euphytica 181:323-339

Galen C, Huddle J, Liscum E (2004) An experimental test of the adaptive evolution of phototropism: blue-light receptors controlling phototropism in Arabidopsis thaliana. Evolution 58:515-523

Galen C, Rabenold JJ, Liscum E (2006) Functional ecology of a blue light photoreceptor: effects of phototropin- 1 on root growth enhance drought tolerance in Arabidopsis thaliana. New Phytol 173:91-99

Gamuyao R, Chin JH, Pariasca-Tanaka J, Pesaresi P, Catausan S, Dalid C, Slamet-Loedin I, Tecson-Mendoza EM, Wissuwa M, Heuer S (2012) The protein kinase Pstoll from traditional rice confers tolerance of phosphorus deficiency. Nature 488:535-539

Ghandilyan A, Ilk N, Hanhart C, Mbengue M, Barboza L, Schat H, Koornneef M, El-Lithy M, Vreugdenhil D, Reymond M, Aarts MG (2009) A strong effect of growth medium and organ type on the identification of QTLs for phytate and mineral concentrations in three Arabidopsis thaliana RIL populations. J Exp Bot 60:1409-1425

Goffinet B, Gerber S (2000) Quantitative trait loci: a meta-analysis. Genetics 155:463-473

Haling RE, Brown LK, Bengough AG, Young IM, Hallett PD, White PJ, George TS (2013) Root hairs improve root penetration, root-soil contact, and phosphorus acquisition in soils of different strength. J Exp Bot 64:3711-3721

Hammond JP, Broadley MR, White PJ, King GJ, Bowen HC, Hayden R, Meacham MC, Mead A, Overs T, Spracklen WP, Greenwood DJ (2009) Shoot yield drives phosphorus use efficiency in Brassica oleracea and correlates with root architecture traits. J Exp Bot 60:1953-1968

Hawkesford M, Horst W, Kichey T, Lambers H, Schjoerring J, Skrumsager Møller I, White P (2012) Chapter 6: Functions of macronutrients. In: Marschner P (ed) Marschner's Mineral Nutrition of Higher Plants, 3rd edn. Academic Press, London, pp 135-189

Hermans C, Hammond JP, White PJ, Verbruggen N (2006) How do plants respond to nutrient shortage by biomass allocation? Trends Plant Sci 11:610-617

Hubert B, Funke GL (1937) The phototropism of terrestrial roots. Biol Jaarboek 4:286-315

Jain A, Poling MD, Karthikeyan AS, Blakeslee JJ, Peer WA, Titapiwatanakun B, Murphy 
AS, Raghothama KG (2007) Differential effects of sucrose and auxin on localized phosphate deficiency-induced modulation of different traits of root system architecture in Arabidopsis. Plant Physiol 144:232-247

Jin K, White PJ, Whalley WR, Shen J, Shi L (2017) Shaping an optimal soil by root-soil interaction. Trends Plant Sci 22:823-829

Kutschera U, Briggs WR (2012) Root phototropism: from dogma to the mechanism of blue light perception. Planta 235:443-452

Lambers H, Clements JC, Nelson MN (2013) How a phosphorus-acquisition strategy based on carboxylate exudation powers the success and agronomic potential of lupines (Lupinus, Fabaceae). Am J Bot 100:263-288

Lambers H, Finnegan PM, Laliberté E, Pearse SJ, Ryan MH, Shane MW, Veneklaas EJ (2011) Phosphorus nutrition of proteaceae in severely phosphorus-impoverised soils: are there lessons to be learned for future crops? Plant Physiol 156:1058-1066

Lapis-Gaza HR, Jost R, Finnegan PM (2014) Arabidopsis PHOSPHATE TRANSPORTER1 genes PHT1;8 and PHT1;9 are involved in root-to-shoot translocation of orthophosphate. BMC Plant Biol 14:334

Liang C, Wang J, Zhao J, Tian J, Liao H (2014) Control of phosphate homeostasis through gene regulation in crops. Curr Opin Plant Biol 21:59-66

Liao H, Yan X, Rubio G, Beebe SE, Blair MW, Lynch JP (2004) Genetic mapping of basal root gravitropism and phosphorus acquisition efficiency in common bean. Funct Plant Biol 31:959-970

Linkohr BI, Williamson LC, Fitter AH, Leyser HM (2002) Nitrate and phosphate availability and distribution have different effects on root system architecture of Arabidopsis. Plant $\mathrm{J}$ 29:751-760

Liu J, Hua W, Hu Z, Yang H, Zhang L, Li R, Deng L, Sun X, Wang X, Wang H (2015) Natural variation in ARF 18 gene simultaneously affects seed weight and silique length in polyploid rapeseed. Proc Natl Acad Sci 112:E5123-E5132

López-Arredondo DL, Leyva-González MA, González-Morales SI, López-Bucio J, HerreraEstrella L (2014) Phosphate nutrition: improving low-phosphate tolerance in crops. Annu Rev Plant Biol 65:95-123

López-Bucio J, Hernández-Abreu E, Sánchez-Calderón L, Nieto-Jacobo MF, Simpson J, Herrera-Estrella L (2002) Phosphate availability alters architecture and causes changes in hormone sensitivity in the Arabidopsis root system. Plant Physiol 129:244-256

López-Bucio J, Hernández-Abreu E, Sánchez-Calderón L, Pérez-Torres A, Rampey RA, Bartel B, Herrera-Estrella L (2005) An auxin transport independent pathway is involved in phosphate stress-induced root architectural alterations in Arabidopsis: identification of 
$B I G$ as a mediator of auxin pericycle cell activation. Plant Physiol 137:681-691

Lynch JP (2007) Roots of the second green revolution. Aust J Bot 55:493-512

Lynch JP (2011) Root phenes for enhanced soil exploration and phosphorus acquisition: tools for future crops. Plant Physiol 156:1041-1049

Mao JL, Miao ZQ, Wang Z, Yu LH, Cai XT, Xiang CB (2016) Arabidopsis ERF1 mediates cross-talk between ethylene and auxin biosynthesis during primary root elongation by regulating ASA1 expression. PLoS Genet 12:e1005760

Meng L, Li H, Zhang L, Wang J (2015) QTL IciMapping: integrated software for genetic linkage map construction and quantitative trait locus mapping in bi-parental populations. Crop J 3:265-279

Metzner R, Eggert A, van Dusschoten D, Pflugfelder D, Gerth S, Schurr U, Uhlmann N, Jahnke S (2015) Direct comparison of MRI and X-ray CT technologies for 3D imaging of root systems in soil: potential and challenges for root trait quantification. Plant Methods $11: 17$

Ming F, Zheng X, Mi G, He P, Zhu L, Zhang F (2000) Identification of quantitative trait loci affecting tolerance to low phosphorus in rice (Oryza Sativa L.). Chinese Sci Bull 45:520 525

Mora-Macías J, Ojeda-Rivera JO, Gutiérrez-Alanís D, Yong-Villalobos L, Oropeza-Aburto A, Raya-González J, Jiménez-Domínguez G, Chávez-Calvillo G, Rellán-Álvarez R, HerreraEstrella L (2017) Malate-dependent Fe accumulation is a critical checkpoint in the root developmental response to low phosphate. Proc Natl Acad Sci 114:E3563-E3572

Müller J, Toev T, Heisters M, Teller J, Moore KL, Hause G, Dinesh DC, Bürstenbinder K, Abel S (2015) Iron-dependent callose deposition adjusts root meristem maintenance to phosphate availability. Dev Cell 33:216-230

Murashige T, Skoog F (1962) A revised medium for rapid growth and bioassays with tobacco tissue cultures. Physiol Plant 15:473-497

Nacry P, Canivenc G, Muller B, Azmi A, Van Onckelen H, Rossignol M, Doumas P (2005) A role for auxin redistribution in the responses of the root system architecture to phosphate starvation in Arabidopsis. Plant Physiol 138:2061-2074

Nakano T, Suzuki K, Fujimura T, Shinshi H (2006) Genome-wide analysis of the ERF gene family in Arabidopsis and rice. Plant Physiol 140:411-432

Nestler J, Wissuwa M (2016) Superior root hair formation confers root efficiency in some, but not all, rice genotypes upon P deficiency. Front Plant Sci 7:1935

Ni JJ, Wu P, Senadhira D, Huang N (1998) Mapping QTLs for phosphorus deficiency tolerance in rice (Oryza sativa L.). Theor Appl Genet 97:1361-1369

Niu YF, Chai RS, Jin GL, Wang H, Tang CX, Zhang YS (2013) Responses of root architecture 
development to low phosphorus availability: a review. Ann Bot 112:391-408

Péreztorres CA, Lópezbucio J, Cruzramírez A, Ibarra-Laclette E, Dharmasiri S, Estelle M, Herrera-Estrella L (2008) Phosphate availability alters lateral root development in Arabidopsis by modulating auxin sensitivity via a mechanism involving the TIR1 auxin receptor. Plant Cell 20:3258-3272

Qiu D, Morgan C, Shi J, Long Y, Liu J, Li R, Zhuang X, Wang Y, Tan X, Dietrich E, Weihmann T, Everett C, Vanstraelen S, Beckett P, Fraser F, Trick M, Barnes S, Wilmer J, Schmidt R, Li J, Li D, Meng J, Bancroft I (2006) A comparative linkage map of oilseed rape and its use for QTL analysis of seed oil and erucic acid content. Theor Appl Genet 114:67-80 Reymond M, Svistoonoff S, Loudet O, Nussaume L, Desnos T (2006) Identification of QTL controlling root growth response to phosphate starvation in Arabidopsis thaliana. Plant Cell Environ 29:115-125

Sánchez-Calderón L, López-Bucio J, Chacón-López A, Gutiérrez-Ortega A, Hernández-Abreu E, Herrera-Estrella L (2006) Characterization of low phosphorus insensitive mutants reveals a crosstalk between low phosphorus-induced determinate root development and the activation of genes involved in the adaptation of Arabidopsis to phosphorus deficiency. Plant Physiol 140:879-889

Shane MW, Lambers H (2005) Cluster roots: a curiosity in context. Plant Soil 274:101-125

Shi L, Shi T, Broadley MR, White PJ, Long Y, Meng J, Xu F, Hammond JP (2013a) Highthroughput root phenotyping screens identify genetic loci associated with root architectural traits in Brassica napus under contrasting phosphate availabilities. Ann Bot 112:381-389

Shi T, Li R, Zhao Z, Ding G, Long Y, Meng J, Xu F, Shi L (2013b) QTL for yield traits and their association with functional genes in response to phosphorus deficiency in Brassica napus. PLoS One 8:e54559

Shi TX, Wang SS, Shi L, Meng JL, Xu FS (2010) Effects of different nitrogen and phosphorus levels on seed yield and quality parameters of double high and double low Brassica napus. Plant Nutr Fert Sci 16:959-964

Silva-Navas J, Moreno-Risueno MA, Manzano C, Pallero-Baena M, Navarro-Neila S, TéllezRobledo B, Garcia-Mina JM, Baigorri R, Gallego FJ, del Pozo JC (2015) D-Root: a system for cultivating plants with the roots in darkness or under different light conditions. Plant J 84:244-255

Silva-Navas J, Moreno-Risueno MA, Manzano C, Téllez-Robledo B, Navarro-Neila S, Carrasco V, Pollmann S, Gallego FJ, del Pozo JC (2016) Flavonols mediate root phototropism and growth through regulation of proliferation-to-differentiation transition. Plant Cell 28:1372-1387 
Svistoonoff S, Creff A, Reymond M, Sigoillot-Claude C, Ricaud L, Blanchet A, Nussaume L, Desnos T (2007) Root tip contact with low-phosphate media reprograms plant root architecture. Nat Genet 39:792-796

Tian J, Wang X, Tong Y, Chen X, Liao H (2012) Bioengineering and management for efficient phosphorus utilization in crops and pastures. Curr Opin Biotechnol 23:866-871

Veneklaas EJ, Lambers H, Bragg J, Finnegan PM, Lovelock CE, Plaxton WC, Price CA, Scheible WR, Shane MW, White PJ, Raven JA (2012) Opportunities for improving phosphorus-use efficiency in crop plants. New Phytol 195:306-320

Walder F, Brulé D, Koegel S, Wiemken A, Boller T, Courty PE (2015) Plant phosphorus acquisition in a common mycorrhizal network: regulation of phosphate transporter genes of the Pht1 family in sorghum and flax. New Phytol 205:1632-1645

Wang S, Basten CJ, Zeng ZB (2011) Windows QTL Cartographer 2.5. Department of Statistics. North Carolina State University, Raleigh, NC

Wang W, Ding GD, White PJ, Wang XH, Jin KM, Xu FS, Shi L (2018) Mapping and cloning of quantitative trait loci for phosphorus efficiency in crops: opportunities and challenges. Plant Soil. https://doi.org/10.1007/s11104-018-3706-6

Wang X, Chen Y, Thomas CL, Ding G, Xu P, Shi D, Grandke F, Jin K, Cai H, Xu F, Yi B, Broadley MR, Shi L (2017) Genetic variants associated with the root system architecture of oilseed rape (Brassica napus L.) under contrasting phosphate supply. DNA Res 24:407417

Wang X, Yan X, Liao H (2010) Genetic improvement for phosphorus efficiency in soybean: a radical approach. Ann Bot 106:215-222

Ward JT, Lahner B, Yakubova E, Salt DE, Raghothama KG (2008) The effect of iron on the primary root elongation of Arabidopsis during phosphate deficiency. Plant Physiol 147:1181-1191

White PJ, Broadley MR, Greenwood DJ, Hammond JP (2005) Genetic modifications to improve phosphorus acquisition by roots. Proceedings 568. York, UK: International Fertiliser Society

White PJ, George TS, Dupuy LX, Karley AJ, Valentine TA, Wiesel L, Wishart J (2013a) Root traits for infertile soils. Front Plant Sci 4:193

White PJ, George TS, Gregory PJ, Bengough AG, Hallett PD, McKenzie BM (2013b) Matching roots to their environment. Ann Bot 112:207-222

Williamson LC, Ribrioux SP, Fitter AH, Leyser HM (2001) Phosphate availability regulates root system architecture in Arabidopsis. Plant Physiol 126:875-882

Xiao G, Qin H, Zhou J, Quan R, Lu X, Huang R, Zhang H (2016) OsERF2 controls rice root growth and hormone responses through tuning expression of key genes involved in 
hormone signaling and sucrose metabolism. Plant Mol Biol 90:293-302

Yang M, Ding G, Shi L, Feng J, Xu F, Meng J (2010) Quantitative trait loci for root morphology in response to low phosphorus stress in Brassica napus. Theor Appl Genet 121:181-193

Yang M, Ding G, Shi L, Xu F, Meng J (2011) Detection of QTL for phosphorus efficiency at vegetative stage in Brassica napus. Plant Soil 339:97-111

Zhang H, Huang Y, Ye X, Xu F (2011) Genotypic variation in phosphorus acquisition from sparingly soluble $\mathrm{P}$ sources is related to root morphology and root exudates in Brassica napus. Sci China Life Sci 54:1134-1142

751

Zhang HW (2009) Study on physiological mechanisms of phosphorus efficiency in Brassica napus. Dissertation, Huazhong Agricultural University

Zhang Y, Thomas CL, Xiang J, Long Y, Wang X, Zou J, Luo Z, Ding G, Cai H, Graham NS, Hammond JP, King GJ, White PJ, Xu F, Broadley MR, Shi L, Meng J (2016) QTL metaanalysis of root traits in Brassica napus under contrasting phosphorus supply in two growth systems. Sci Rep 6:33113

Zhu J, Kaeppler SM, Lynch JP (2005a) Mapping of QTLs for lateral root branching and length in maize (Zea mays L.) under differential phosphorus supply. Theor Appl Genet 111:688695

Zhu J, Kaeppler SM, Lynch JP (2005b) Topsoil foraging and phosphorus acquisition efficiency in maize (Zea mays). Funct Plant Biol 32:749-762 
Table 1 Means, ranges and coefficients of variation $(\mathrm{CVs})$ of the relative root traits in the parental lines and the BnaTNDH mapping population in the 'agar' system and the 'pouch and wick' system

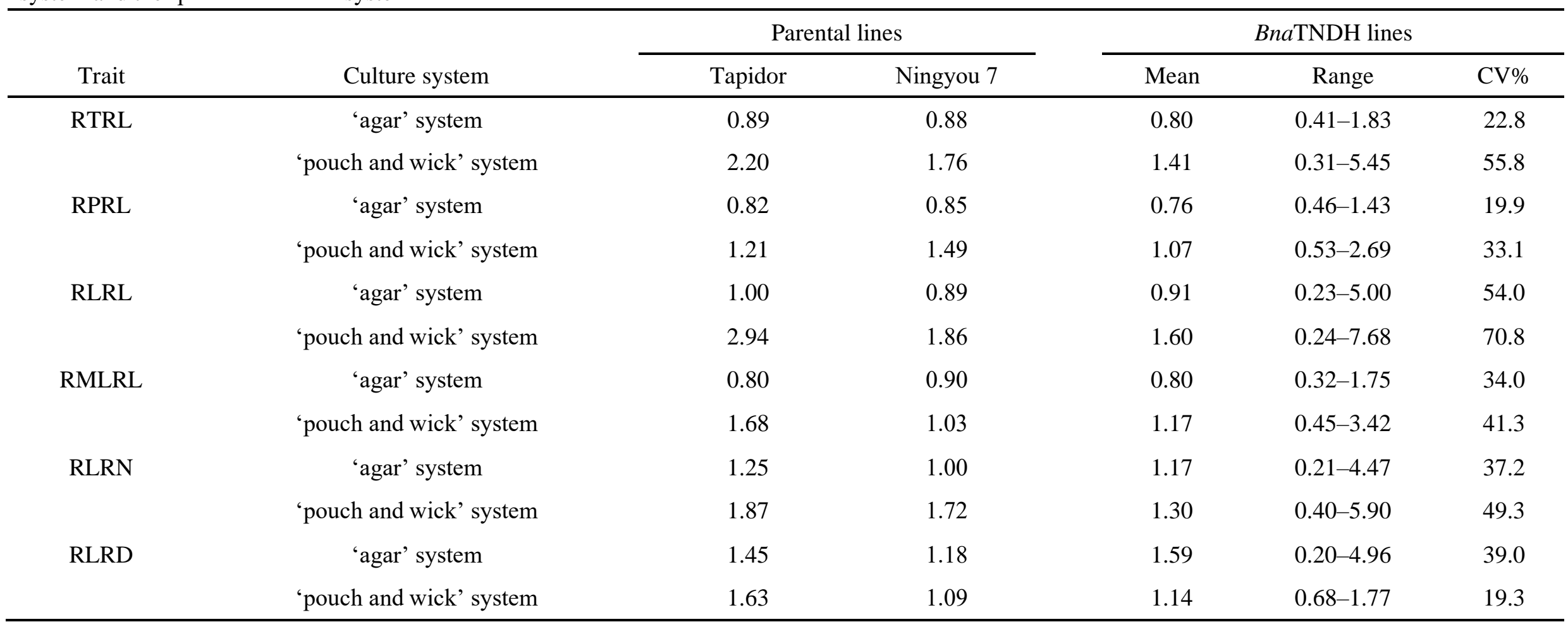


Table 2 Co-located QTLs for the relative traits in the 'agar' system and the 'pouch and wick' system with the seed yield and yield-related traits in the field trials

\begin{tabular}{|c|c|c|c|c|c|}
\hline \multirow[b]{2}{*}{$\mathrm{z}$} & \multicolumn{3}{|c|}{ QTLs for the relative traits } & \multicolumn{2}{|c|}{ QTLs for the seed yield and yield-related traits in the field trials } \\
\hline & Culture system & QTL name & Confidence interval (cM) & QTL name & Confidence interval (cM) \\
\hline \multirow[t]{3}{*}{ A04 } & 'agar' system & RLRN A04b & $10.0-12.3$ & SW OP1 A04a & $9.9-14.8$ \\
\hline & & & & SW_LP2_A04b & $9.0-14.8$ \\
\hline & & & & SW_OP2_A04b & $7.4-15.4$ \\
\hline \multirow[t]{9}{*}{ A09 } & & RLRL_A09 & $52.5-59.4$ & BN_OP3_A09b & $45.8-52.6$ \\
\hline & & RSDW_A09a & $124.4-129.4$ & SY_LP1_A09a & $124.3-129.4$ \\
\hline & & & & PH_OP1_A09a & $124.5-129.4$ \\
\hline & & & & FBH_OP2_A09a & $128.8-134.3$ \\
\hline & & Cluster1 & $129.5-131.3$ & SY_LP1_A09b & $130.9-135.4$ \\
\hline & & & & FBH_OP2_A09a & $128.8-134.3$ \\
\hline & & & & PH_OP2_A09a & $129.4-132.3$ \\
\hline & & & & SY_OP2_A09 & $130.4-135.4$ \\
\hline & & Cluster2 & $135.9-138.1$ & FBH_OP3_A09 & $137.3-139 . z$ \\
\hline C09 & & RRFW_C09b & $61.4-64.2$ & RBH_OP3_C09a & $61.4-64.3$ \\
\hline A03 & 'pouch and wick' system & RPRL_A03 & $3.1-23.6$ & SW_LP1_A03a & $20.4-27.8$ \\
\hline & & & & BN_OP1_A03 & $0-8.9$ \\
\hline
\end{tabular}

The QTLs for the seed yield and yield-related traits in the three field trials were denominated as "trait+P treatment+trial number+chromosome+the serial letter". Height to the first primary branch (cm; FBH), plant height (cm; PH), relative first primary branch height (the ratio of FBH to PH; RBH), number of primary branches per plant (N; BN), seed weight of 1,000 seeds (g per 1000 seeds; SW), seed yield per hectare (kg.ha-1; SY). LP, a low phosphorus supply. OP, an optimal phosphorus supply 

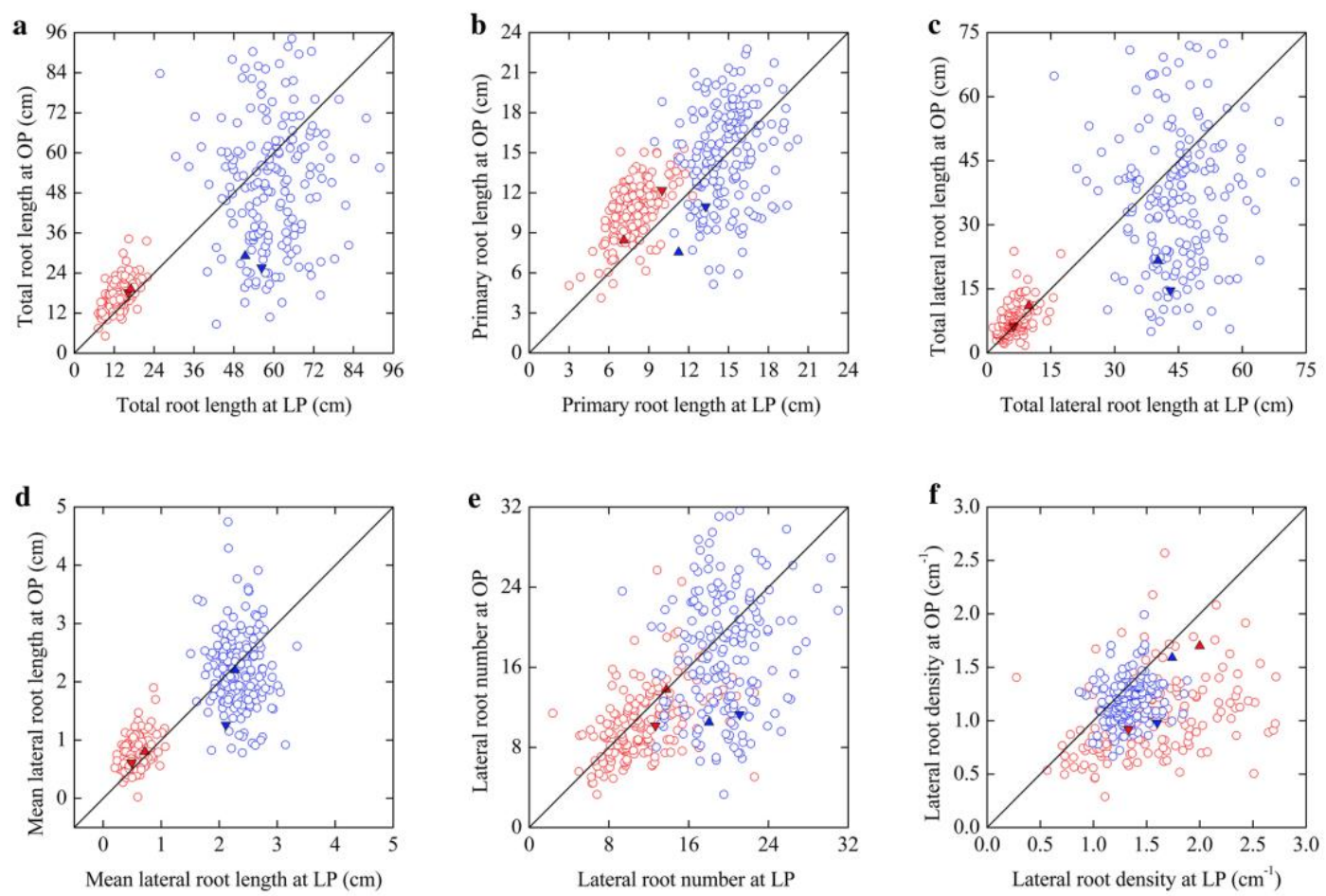

Fig. 1 Variation in total root length (a), primary root length (b), total lateral root length (c), mean lateral root length (d), lateral root number (e), lateral root density (f) of the BnaTNDH mapping population in the 'agar' and 'pouch and wick' systems. The open red circle and open blue circle represent the DH lines in the 'agar' system and the 'pouch and wick' system, respectively. The solid red downtriangle and solid red uptriangle represent Tapidor and Ningyou 7 in the 'agar' system, respectively. The solid blue downtriangle and solid blue uptriangle represent Tapidor and Ningyou 7 in the 'pouch and wick' system, respectively. The continuous line represents the $1: 1$ line 

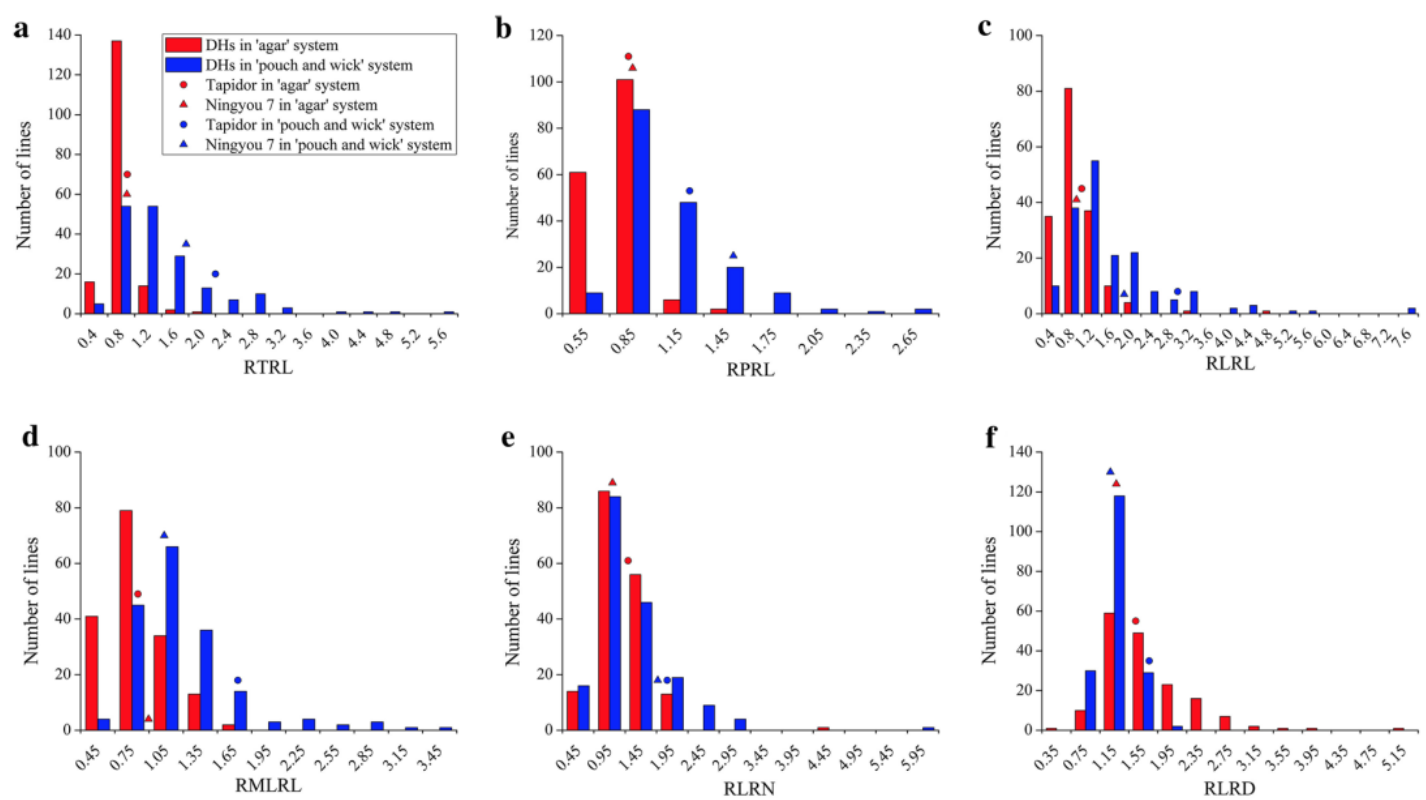

779

Fig. 2 Frequency distribution of relative root traits of the BnaTNDH mapping population in the 'agar' (red bar) and 'pouch and wick' (blue bar) systems. RTRL (a), relative total root length; RPRL (b), relative primary root length; RLRL (c), relative total lateral root length; RMLRL (d), relative mean lateral root length; $\operatorname{RLRN}(\mathbf{e})$, relative lateral root number; RLRD (f), relative lateral root density. The solid red circle and solid red uptriangle represent Tapidor and Ningyou 7 in the 'agar' system, respectively. The solid blue circle and solid blue uptriangle represent Tapidor and Ningyou 7 in the 'pouch and wick' system, respectively 


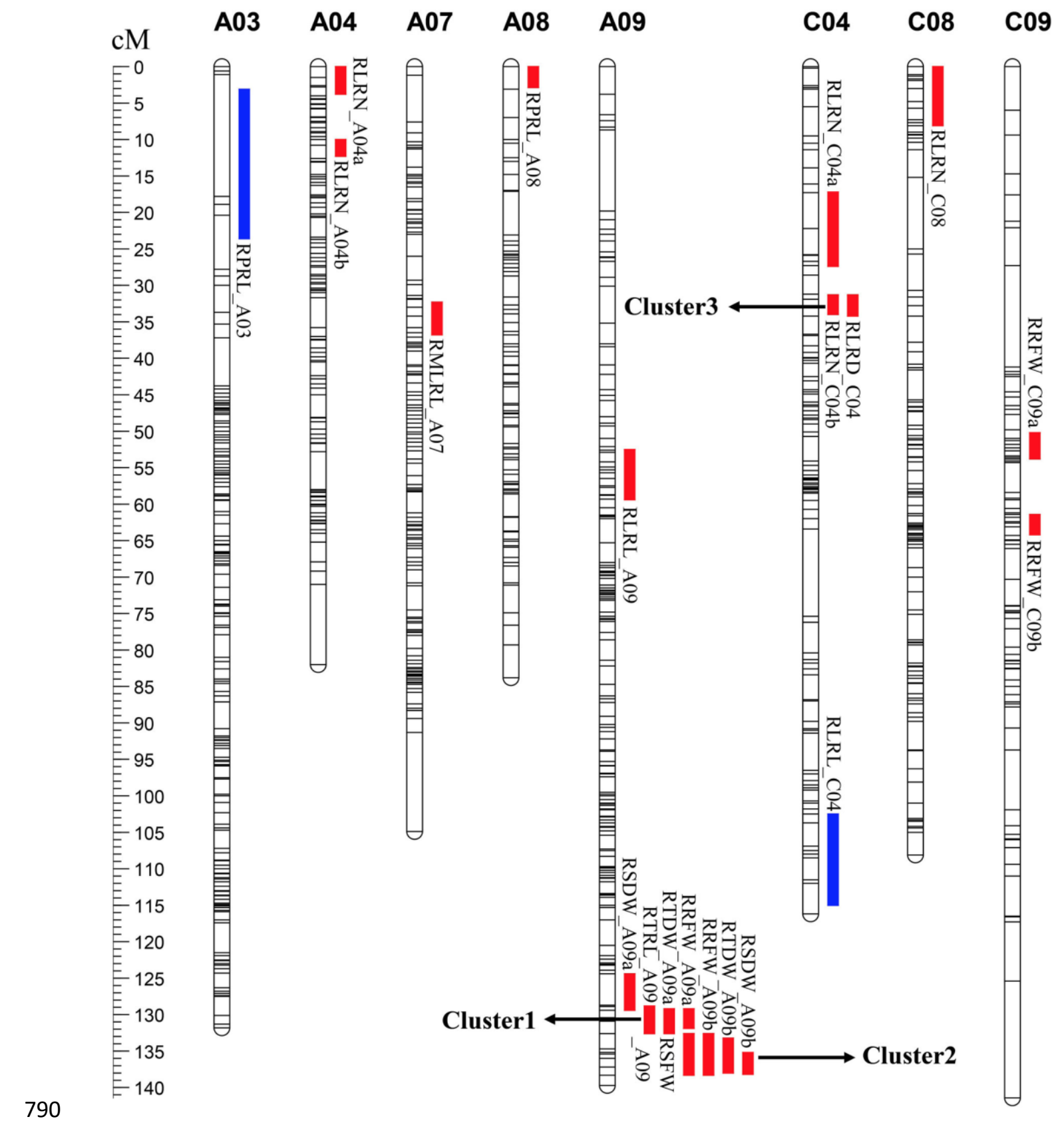

792 Fig. 3 Locations of QTLs for relative root traits and relative biomass traits in the BnaTNDH 793 mapping population in the 'agar' and 'pouch and wick' systems. QTLs are indicated on the right 794 side of each chromosome. The red and blue bars denote the QTLs identified in the 'agar' system 795 and the 'pouch and wick' system, respectively. The QTL confidence intervals are set as the map 796 interval corresponding to a 2-LOD decline on either side of the LOD peak. RTRL, relative total 797 root length; RPRL, relative primary root length; RLRL, relative total lateral root length; 798 RMLRL, relative mean lateral root length; RLRN, relative lateral root number; RLRD, relative 799 lateral root density; RTDW, relative total dry weight; RSDW, relative shoot dry weight; RSFW, 800 relative shoot fresh weight; RRFW, relative root fresh weight 


\section{Supplementary Figure Legends}

Supplementary Fig. 1 The plasticity of root traits of the BnaTNDH mapping population in response to phosphorus deficiency in the 'agar' and 'pouch and wick' systems. a, total root length (TRL); b, primary root length (PRL); $\mathbf{c}$, total lateral root length (LRL); d, mean lateral root length (MLRL); e, lateral root number (LRN); f, lateral root density (LRD). Boxes represent the mid two quartiles with the median and mean drawn. Whiskers are the $95 \%$ confidence limits plus extremes

Supplementary Fig. 2 Location of QTLs for TRL (total root length), PRL (primary root length), LRL (total lateral root length), MLRL (mean lateral root length), LRN (lateral root length), LRD (lateral root density) and its relative traits. The red bar above the chromosome denotes the QTL identified at a low P supply. The green bar below the chromosome denotes the QTL identified at an optimal P supply. The purple bar inside the chromosome denotes the QTL for relative root trait. The red star indicates that the QTL for a root trait is co-located with the QTL for its relative trait

Supplementary Fig. 3 The illumination of roots altered the response of root architecture to phosphate deprivation in Arabidopsis thaliana. Col-0 seedlings were grown at a low (-P, $0 \mathrm{mM}$ ) and an optimal P supply ( + P, $0.625 \mathrm{mM})$ with the root exposed to light (LGR, light-grown roots) or in darkness (DGR, dark grown roots) in an illuminated culture room with $16 \mathrm{~h}$ photoperiod of approximately $300-320 \mu \mathrm{mol} \mathrm{m}-2 \mathrm{~s}-1$, temperature at $18-24^{\circ} \mathrm{C}$ and a relative humidity of $65-$ $80 \%$ for 21 days. Scale bar $=2 \mathrm{~cm}$

Supplementary Fig. 4 The illumination of roots altered the response of root architecture to phosphate deprivation in Brassica napus. Tapidor and Ningyou 7 seedlings were grown at a low (-P, $0 \mathrm{mM})$ and an optimal P supply (+P, $0.625 \mathrm{mM})$ with the root exposed to light (LGR, light-grown roots) or in darkness (DGR, dark grown roots) in an illuminated culture room with $16 \mathrm{~h}$ photoperiod of approximately $300-320 \mu \mathrm{mol} \mathrm{m}-2 \mathrm{~s}-1$, temperature at $18-24{ }^{\circ} \mathrm{C}$ and a relative humidity of $65-80 \%$ for 9 days. Scale bar $=3 \mathrm{~cm}$

Supplementary Fig. 5 Total root length (a), primary root length (b), total lateral root length (c), mean lateral root length (d), lateral root number (e), lateral root density (f) of Tapidor and Ningyou 7 seedlings grown at a low (-P, $0 \mathrm{mM})$ and an optimal P supply $(+\mathrm{P}, 0.625 \mathrm{mM})$ with the root exposed to light (LGR, light-grown roots) or in darkness (DGR, dark grown roots). Data are shown as mean $\pm \mathrm{SD}(n=3-6)$. Asterisks indicate statistically significant differences between $-\mathrm{P}$ and $+\mathrm{P}\left({ }^{*}, P<0.05 ; * *, P<0.01\right)$ according to Student's $t$-test. 